Supplementary Information to:

\title{
Dynamics and Mechanisms of Exfoliated Black Phosphorus Sublimation
}

\author{
Matthieu Fortin-Deschênes, ${ }^{1}$ Pierre Levesque, ${ }^{2}$ Richard Martel, ${ }^{2}$ Oussama Moutanabbir ${ }^{1}$ \\ ${ }^{1}$ Department of Engineering Physics, École Polytechnique de Montréal, C. P. 6079, Succ. \\ Centre-Ville, Montréal, Québec H3C 3A7, Canada \\ ${ }^{2}$ Département de Chimie, Université de Montréal, 2900 boulevard Edouard Montpetit, Montréal, \\ Canada
}



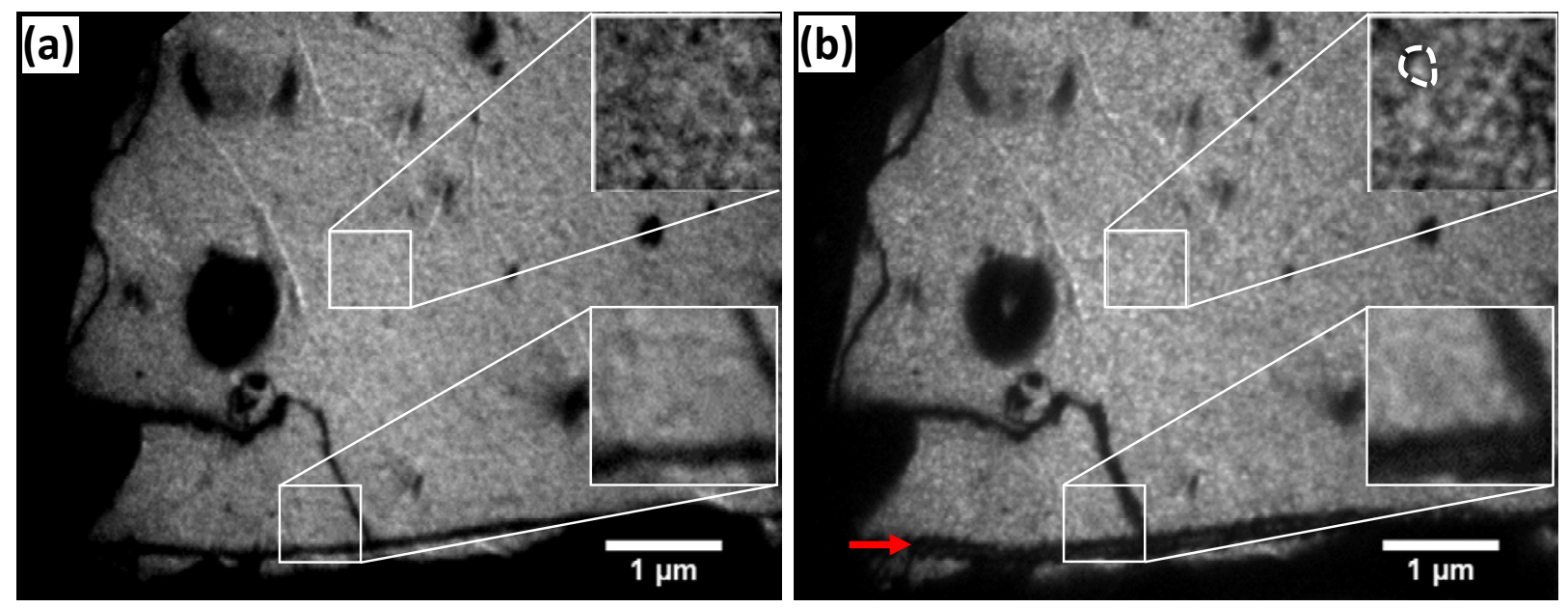

Figure S1 Initial stage of sublimation. LEEM images recorded at $375^{\circ} \mathrm{C}$ : (a) Before first signs of sublimation; (b) After 366 seconds. The image in (b) shows many holes of similar size on the surface. Inset (top) shows a zoom where multiple holes can be seen, with one highlighted in dashed white line. Red arrow highlights the sublimation at edge. Inset (bottom) show a zoom on edge sublimation. 



Figure S2 (a), (b), and (c) LEEM snapshots showing sublimation near a defect across several layers. The yellow dot in (a) shows the nucleus of the first hole on the top layer. Arrows in (c) indicate the concentric holes on the bottom layers. (d), (e), and (f) LEEM images highlighting the sublimation at a linear defect extending trough multiple layers. The yellow line in (d) indicates the presence of a linear defect on the first layer. Arrows in (c) show the edges of the holes on multiple layers. 

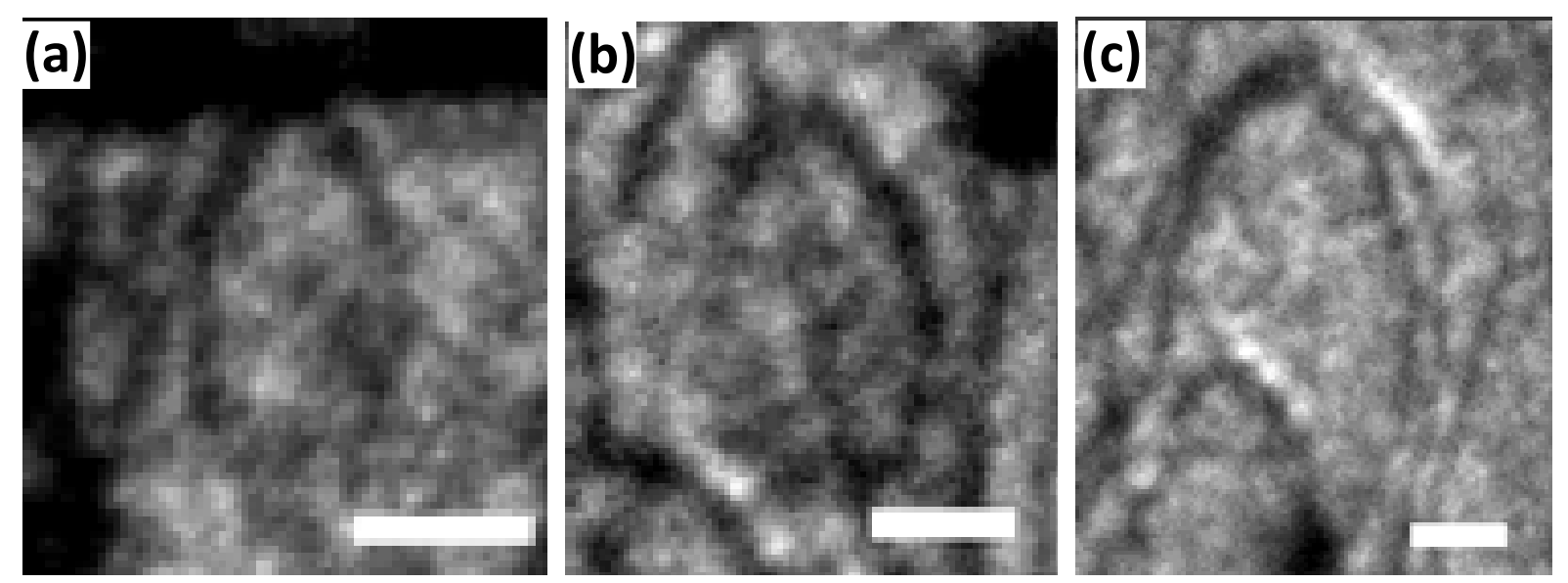

Figure S3 LEEM images of three different holes taken at $495^{\circ} \mathrm{C}$ showing that the long edges are usually rounded and that some hole tips are not sharp. Roundness of hole tip in (c) can be attributed to step bunching. Scale bars are $150 \mathrm{~nm}$.

\section{LEEM/LEED rotational angle calibration}

As explained in the main manuscript, we observed holes with a long axis aligned with the [100] direction, which contradicts the observations from Liu et al. ${ }^{1}$ where the holes were observed along the [001] direction. Because both observations are made with electron microscopes, which use magnetic lenses that can rotate the image, it is possible that this contradiction comes from the misalignment between diffraction and imaging modes in either one of the systems. Therefore, we verified the calibration of the rotational angle between LEEM and LEED modes used in our study using two independent methods.

The first calibration is shown in Figure S4. A graphene covered Ge(001) sample is used. The $\mathrm{Ge}(001)$ forms $\{107\}$ facets underneath the graphene. Those facets form long triangular prismlike structures along the $<100>$ directions of $\operatorname{Ge}(001)^{2}$. The $(0,0)$ spots of the $\{107\}$ facets can be seen with LEED. The directions between the $(0,0)$ spot of $\operatorname{Ge}(001)$ and the $(0,0)$ spots of the facets in the LEED pattern should be perpendicular to the associated triangular prism structures (or parallel to projection of the normal of the $\{107\}$ planes on the (001) surface). Dark-field of the facets show no rotation between LEEM and LEED modes. AFM of the graphene covered $\mathrm{Ge}(001)$ surface (reproduced with permission) ${ }^{2}$ illustrate the triangular prism structures. 


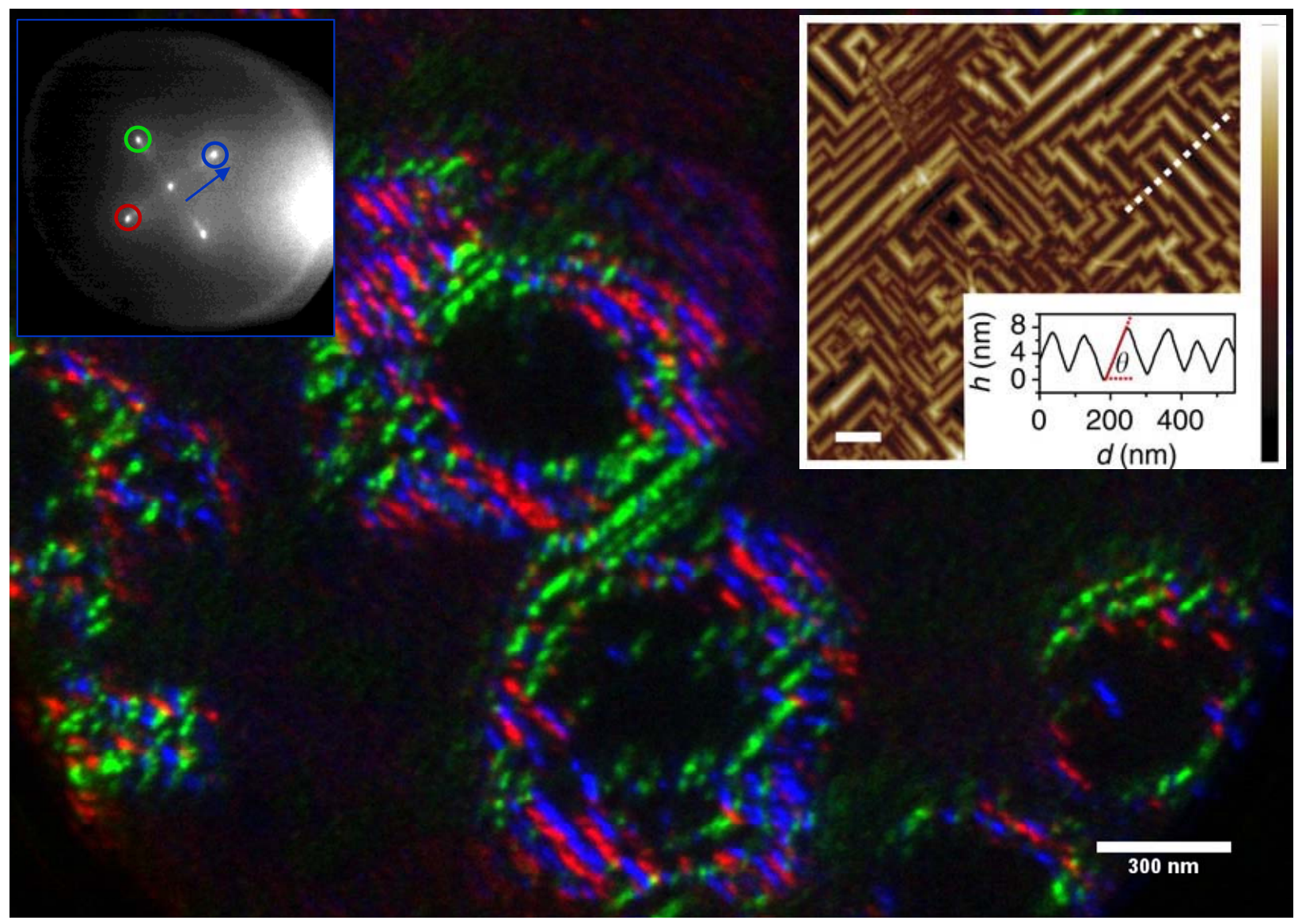

Figure S4 Superposition of 3 LEEM dark-field images of Ge(107) facets on graphene covered $\mathrm{Ge}(001)$. Inset (top left) shows the associated LEED pattern with red, blue and green circles marking the LEED spots used for the dark-field images. Inset (top right), reproduced with permission, shows an AFM image (scale bar 200nm; height scale bar $11.8 \mathrm{~nm}$ ) of the facets forming long triangular prism like structures

The second calibration was made using $\mathrm{Si}(110)$, which has a rectangular surface lattice, therefore a rectangular LEED pattern. We used photolithography to pattern lines in a specific direction which we observed in LEEM mode and compared to the LEED pattern. The in plane orientation of the $\mathrm{Si}(110)$ wafer was calibrated with XRD after the lithography process. 

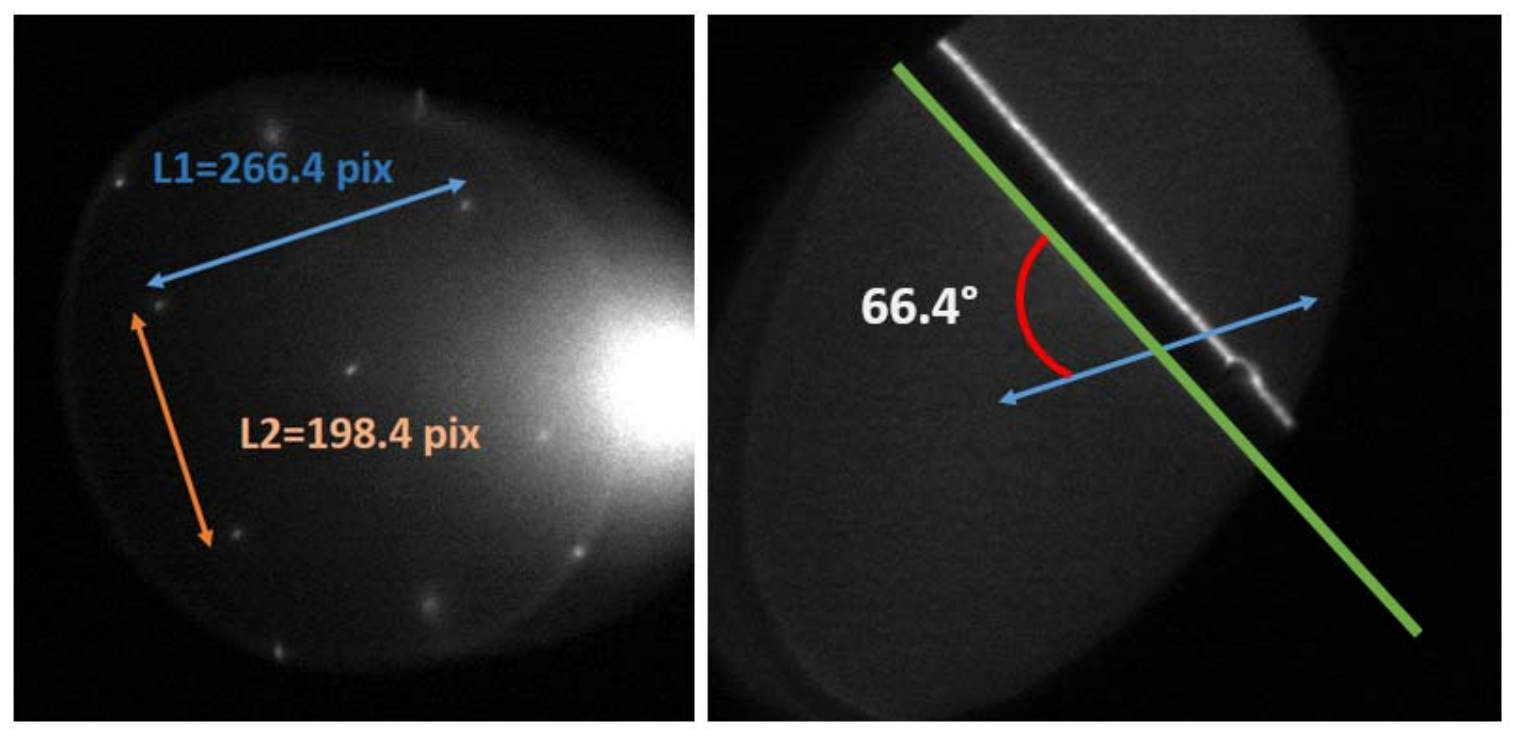

Figure S5 LEED pattern (on the left) and corresponding LEEM image (on the right) of Si(110) calibration sample. In the LEED pattern, L1 corresponds to the [110] direction and L2 to the [001] direction. The green line was determined by XRD to be at $68.6^{\circ}$ from the [110] direction

Using a previous calibration on a graphene sample, we determine that the scale in LEED is $1.96 \times 10^{7} \mathrm{~m}^{-1} /$ pixel. Therefore, $L 1$ corresponds to a lattice parameter of $3.83 \AA$ and $L 2$ to a lattice parameter of $5.15 \AA$. We conclude that L1 is parallel to the [110] direction and $L 2$ is parallel to the [001] direction of silicon, if LEED and LEEM are correctly aligned. Note that here, $3.83 \AA$ corresponds to $\sqrt{2} / 2 a_{S i}$, where $a_{S i}=5.43 \AA$. The $5 \%$ error between $5.15 \AA$ and $5.43 \AA$ can be attributed to beam deformation (we see that the Ewald sphere which should appear circular is a bit elongated in the direction parallel to L2, which leads to a higher L2, therefore to a shorter lattice parameter). We see in Fig S4 (right) that there is a $66.4^{\circ}$ angle between the [110] direction and the green line (etched pattern made by photolithography).

We then use XRD to determine the orientation of the etched lines on $\mathrm{Si}(110)$ as shown in Fig. S6. We fix the Bragg condition at $2 \theta=88.0^{\circ}$ to detect only the $\{422\}$ planes $(\lambda=1.541 \AA)$ and we fix $\omega=14.0^{\circ}$ (incident $x$-ray angle to the surface) to measure only the 8 planes making a

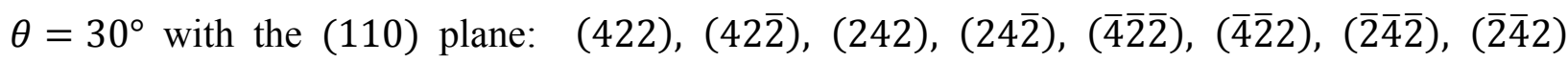
and measure the diffracted signal for $\phi=0$ to $\phi=360^{\circ}$. We know that if the [110] direction is at $\phi=0$ (parallel to the lithography lines), then we should measure 4 peaks at: $54.74^{\circ}, 125.26^{\circ}$, $234.74^{\circ}$ and $305.26^{\circ}$, which correspond to the angle between the [110] direction and the projection of the normal of the $\{422\}$ planes on the (110) plane. This means that the [110] 
bisects a $109.5^{\circ}$ angle. $\left(305.26^{\circ}\right.$ to $\left.54.74^{\circ}\right)$ or $\left(125.26^{\circ}\right.$ to $\left.234.74^{\circ}\right)$. The deviation from those angles is the angle between the [110] direction and the lithography lines.

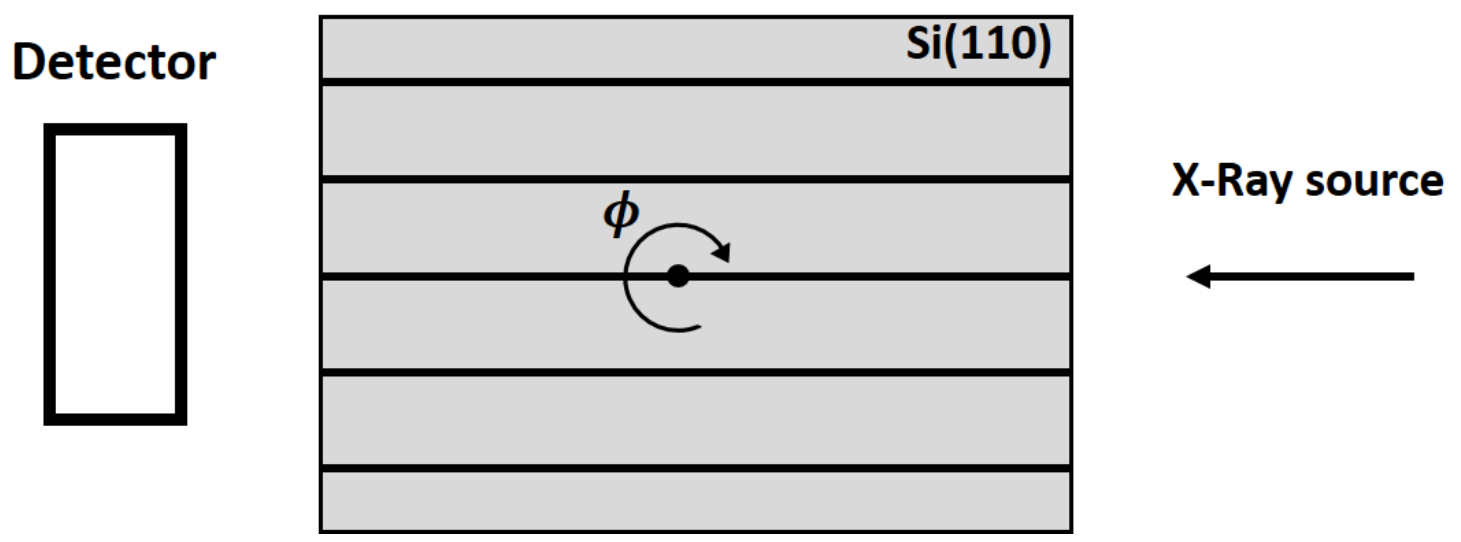

Figure S6 Schematic illustration of experiment used to confirm the alignment of our LEEM/LEED setup.

The measured peak positions (shown on Fig. S7) are at: $13.1^{\circ}, 115.0^{\circ}, 194.4^{\circ}$ and $304.4^{\circ}$, which might look strange at first. However, the $115.0^{\circ}$ peak has a large shoulder so we choose to ignore because this position shift is likely due to an alignment error. The $109.5^{\circ}$ angle is therefore between the 2 last peaks so the [110] direction is at $\phi=(194.4+304.4) / 2=249.4^{\circ}$ or $\phi=69.4$. Considering only the first peak at $13.1^{\circ}$, we find $\phi=67.8^{\circ}$ with a mean value of $\phi=68.6^{\circ}$. This is in good agreement with the LEEM and LEED measurement, where we find $\phi=66.4^{\circ}$, meaning that LEEM and LEED are well aligned (or have a $180^{\circ}$, which is not relevant here considering that $\mathrm{bP}$ has a $180^{\circ}$ rotational symmetry). Considering only this measurement, there is also the possibility that they are misaligned by $\alpha= \pm(180-$ $(66.4+68.6))= \pm 45.0^{\circ}$. This is however inconsistent with our other measurements on surfaces with 4-fold symmetry ${ }^{2}$. 


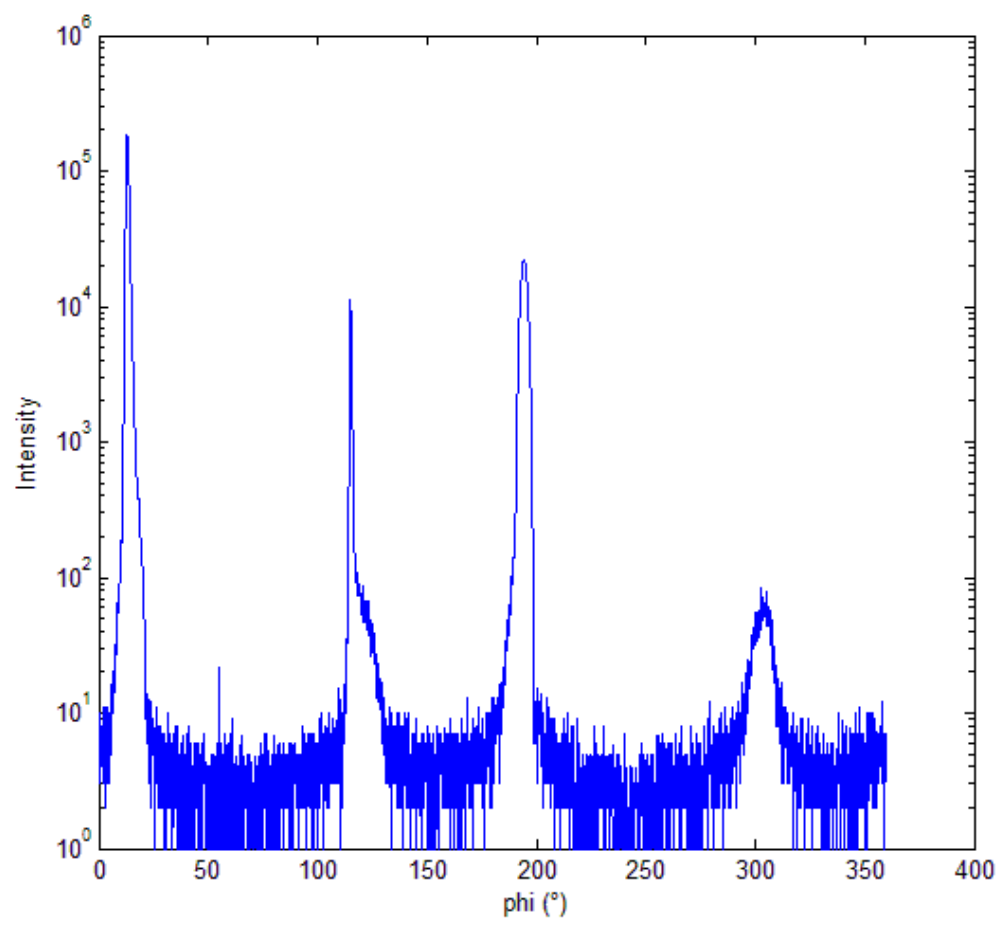

Figure S7 Measured XRD intensity vs rotational angle $\phi$ (configuration shown in Fig.S6) for the $\{422\}$ planes making a $30^{\circ}$ angle with the (110 plane)

\section{Analytical description of the sublimation of 2D-bP materials}

This section describes the analytical model used to determine the total sublimation flux $F$ of $P_{2}$ molecules from a multilayer bP film and its dependence on surface defect density, $\rho_{d}$, hole expansion velocity, $v$, and time, $t$. To simplify the problem, we consider circular holes instead of anisotropic holes. The exact hole shape should not affect the sublimation flux general behaviour with respect to $\rho_{d}, v$ and $t$. We consider a continuous film (no edges) of multilayer BP with point defects randomly distributed according to a Poisson process. The hole nucleation is treated as follows: At $t<0$, the temperature is too low to allow for sublimation. At $t=0$, the temperature is abruptly raised at a temperature where bP sublimates. As shown in Fig. S1, holes on the top layer nucleate all at the same time (therefore on defects that are already present). For the bP layers underneath, we consider also randomly distributed defects (distributed according to a Poisson process). Holes nucleate as soon as the defects are exposed to vacuum (by sublimation of the top layer). This assumption is not entirely true, as we usually observe a small delay between the sublimation of the top layer and the hole nucleation on the layer underneath (maybe due to a small activation energy for nucleation at defects, or a slower expansion velocity in the 
early stage of hole expansion). Nevertheless, this assumption seems in rather good agreement with the observations shown in Fig. S2. The other assumption is that the defects are randomly distributed, which is again not entirely true, as shown in Fig. S2, because defects that extend on multiple layers are observed in our sample. However, those defects are likely due to exfoliation and sample preparation and would likely not be present on an epitaxially grown film.

The sublimation flux for circular holes nucleating at $\mathrm{t}=0$ is given by:

$$
F_{1}=l_{1} * v * \rho_{S} * X_{1}
$$

, where $\rho_{S}$ is the surface atomic density (atoms $\left./ \mathrm{m}^{2}\right), l_{1}$ is the total amount of edges per unit surface (neglecting coalescence), $X_{1}$ is a correction term for coalescence and $v$ is the hole expansion velocity. We can write $l_{1}$ :

$$
l_{1}=\rho_{d} * 2 * \pi *(v * t)
$$

, where $(v * t)$ is the hole radius expanding with time $t$. To correct for the coalescence of holes (which reduces the amount of edges), we multiply $l_{1}$ by the probability that a random point on the surface has no hole nuclei within a distance $v * t$ around it (corresponding to the fraction of $l_{1}$ which has not coalesced) and we get:

$$
F_{1}=\rho_{\mathrm{S}}\left(2 \pi \mathrm{v}^{2} \mathrm{t} \rho_{\mathrm{d}}\right) \mathrm{e}^{-\pi \rho_{\mathrm{d}} \mathrm{v}^{2} \mathrm{t}^{2}} .
$$

Then for the second layer, we consider that a defect is exposed at $t=t_{0}$ and that a hole immediately nucleates. The hole nucleation rate on the second layer is given by:

$$
\frac{d n_{2}}{d t}=-\rho_{d} * \frac{d S_{1}}{d t}
$$

, where $\frac{d S_{1}}{d t}$ is the rate at which the first layer disappears. Assuming that the radius of the holes is given by:

$$
r=v *\left(t-t_{0}\right) * \Theta\left(t-t_{0}\right)
$$

, where $\Theta\left(t-t_{0}\right)$ is the Heaviside function, we have:

$$
l_{2}=\left.\int_{0}^{t} \frac{d n_{2}}{d t}\right|_{t=t_{0}} * 2 * \pi * v *\left(t-t_{0}\right) * \Theta\left(t-t_{0}\right) d t_{0} .
$$

With a correction factor for coalescence given by:

$$
X_{2}=e^{-A_{2}}
$$


, where $A_{2}$ is the total area of the holes, neglecting coalescence:

$$
A_{2}=\left.\int_{0}^{t} \frac{d n_{2}}{d t}\right|_{t=t_{0}} \pi * v^{2} *\left(t-t_{0}\right)^{2} \times \Theta\left(t-t_{0}\right) d t_{0} .
$$

Solving the integrals, we find:

$$
F_{2}=v \rho_{S} \sqrt{\pi \rho_{d}}\left(2 t v \sqrt{\pi \rho_{d}}-\sqrt{\pi} \operatorname{erf}\left(t v \sqrt{\pi \rho_{d}}\right)\right) e^{Y}
$$

with

$$
Y=1-t \pi v \sqrt{\rho_{d}} \operatorname{erf}\left(t v \sqrt{\pi \rho_{d}}\right)+\pi \rho_{d} v^{2} t^{2}-e^{-t^{2} \pi \rho_{d} v^{2}}
$$

For the layers below, it is not straight forward to derive an analytical solution. However, using the solution of the first 2 layers, we can calculate numerically the sublimation flux for all layers depending on $\rho_{d}, v$ and $t$. We use the following relations:

$$
F_{i}=v * \rho_{S} * l_{i} * e^{-A_{i}}
$$

with

$$
l_{i}=\int_{0}^{t}-\left.\rho_{d} * \frac{d S_{i-1}}{d t}\right|_{t=t_{0}} * 2 * \pi * v *\left(t-t_{0}\right) * \Theta\left(t-t_{0}\right) d t_{0}
$$

and

$$
A_{i}=\int_{0}^{t}-\left.\rho_{d} * \frac{d S_{i-1}}{d t}\right|_{t=t_{0}} * \pi * v^{2} *\left(t-t_{0}\right)^{2} * \Theta\left(t-t_{0}\right) d t_{0}
$$

, where

$$
\frac{d s_{i}}{d t}=l_{i} * v * e^{-A_{i}}
$$

Considering that the total sublimation flux is given by:

$$
F(t)=\sum_{i} F_{i}(t)
$$

, we get the behavior shown in Fig. S8 for the total sublimation flux. This is achieved using the analytical solution for the first 2 layers and numerical solutions for 298 other layers below (equivalent to a 300 layers BP thin film). 


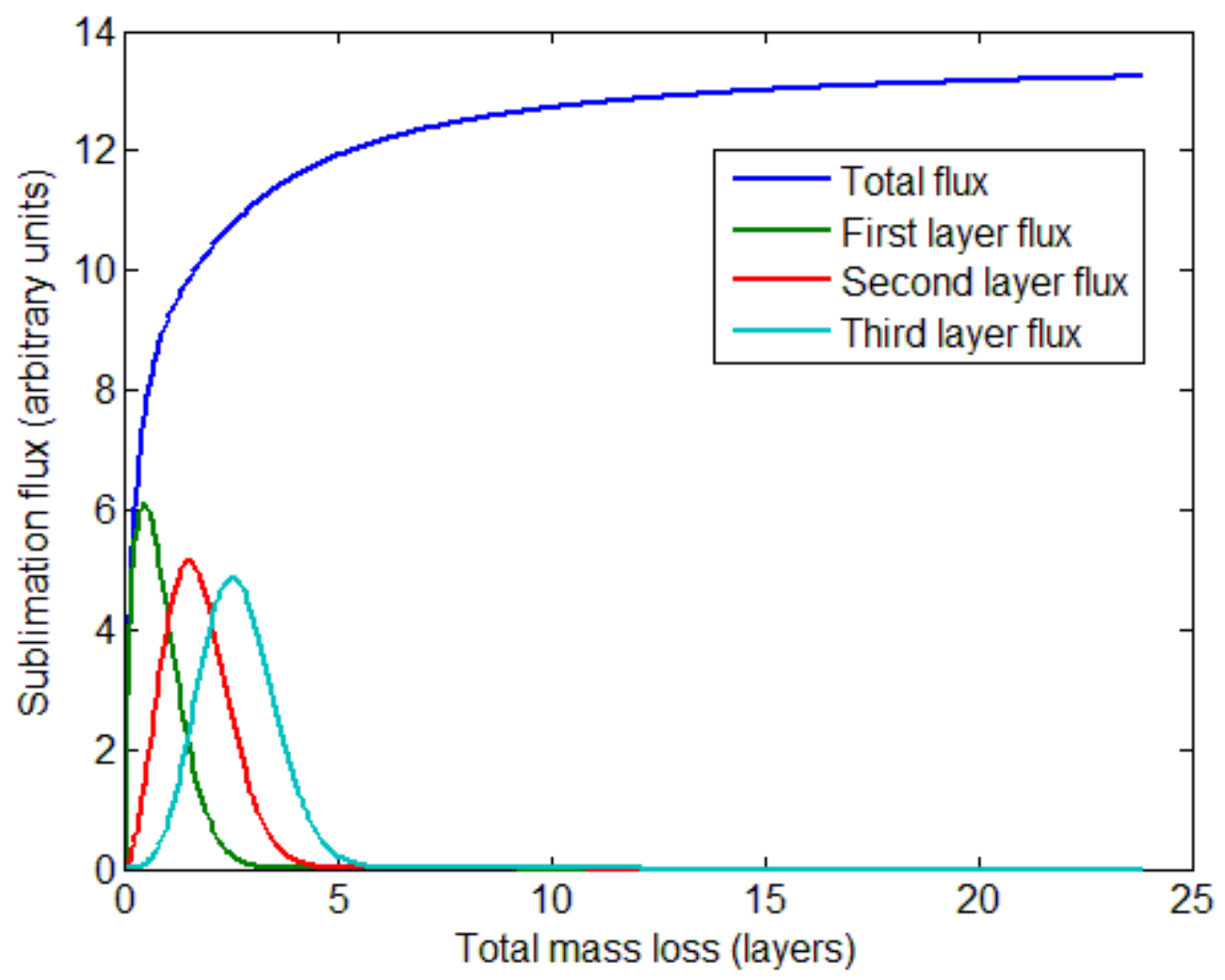

Figure S8 Sublimation flux for a 300 layers-thick bP film according to equations (3), (8), (9-11) and (14)

To determine the dependence of the sublimation flux on $\rho_{d}$ and $v$, we take the slope of the total sublimation flux $F$ vs. $\rho_{d}$ or $v$ in log-log scale at fixed mass loss (fixed time would yield different exponents in the early stage of sublimation because steady-state is not yet reached). An example for $\rho_{d}$ is shown on Figure S9. 


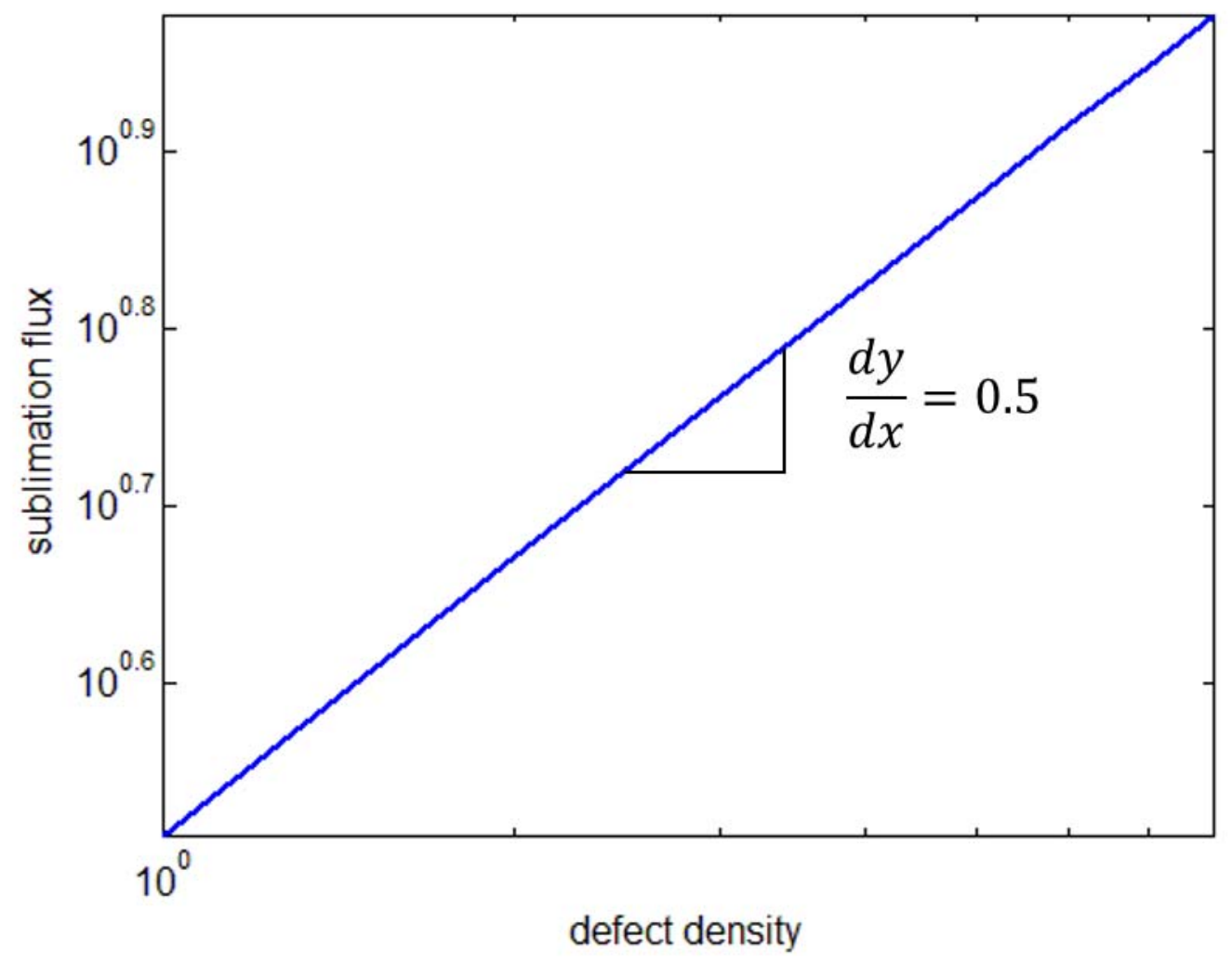

Figure S9 Sublimation flux vs. defect density according to equation (14) when steady-state is reached. The 0.5 slope correspond to the $\rho_{d}^{0.5}$ dependency

Finally, to determine if this model leads to a layer-by-layer or multilayer sublimation, we simulate the RHEED intensity vs. time with the equation:

$$
I_{R H E E D}=\left(\sum_{n=1}^{N}(-1)^{n} \theta_{n}\right)^{2}
$$

If the sublimation is layer by layer, we will see RHEED oscillations and if it is multilayer, the intensity will drop exponentially to 0 . 


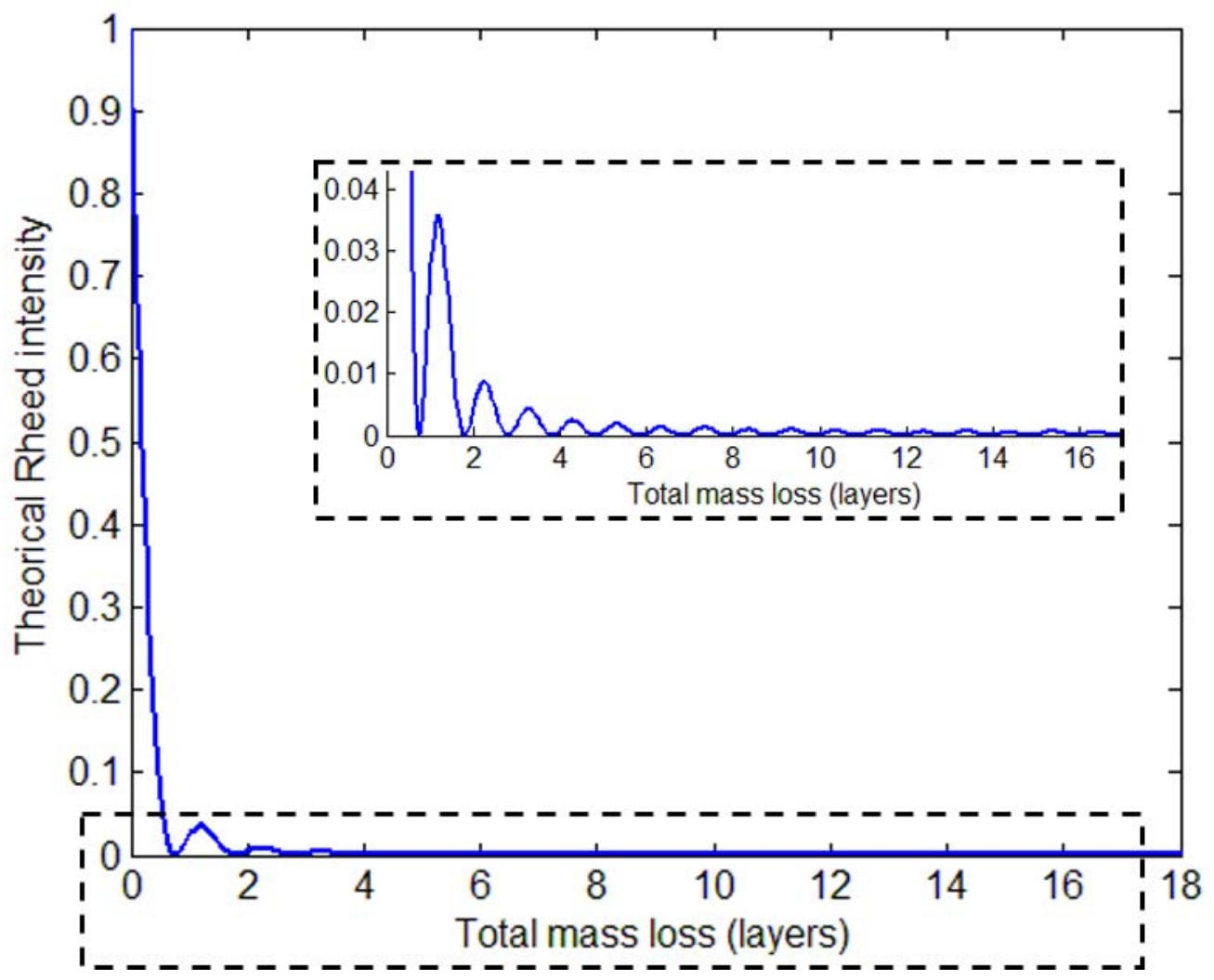

Figure S10 Simulated RHEED intensity vs. time according to equations (14) and (15)

We see a weak layer-by-layer behavior which fades quickly after a few layers, consistent with LEEM observations, where many layers are exposed at the same time. We also note that the observed delay between the exposition of a nucleation site and the nucleation of the hole will lead to a more pronounced layer-by-layer behavior. However, the defects that are extending on multiple layers reduce de layer-by-layer behavior. 


\section{References}

1. Liu, X.; Wood, J. D.; Chen, K.-S.; Cho, E.; Hersam, M. C., In Situ Thermal Decomposition of Exfoliated Two-Dimensional Black Phosphorus. The Journal of Physical Chemistry Letters 2015, 6, 773-778.

2. Jacobberger, R. M.; Kiraly, B.; Fortin-Deschenes, M.; Levesque, P. L.; McElhinny, K. M.; Brady, G. J.; Rojas Delgado, R.; Singha Roy, S.; Mannix, A.; Lagally, M. G.; Evans, P. G.; Desjardins, P.; Martel, R.; Hersam, M. C.; Guisinger, N. P.; Arnold, M. S., Direct oriented growth of armchair graphene nanoribbons on germanium. Nat Commun 2015, 6. 8006 